\title{
An object-oriented and colored timed Petri Net Model for automated Interbay material handling system of semiconductor wafer fab
}

\author{
Lihui $\mathrm{WU}^{1, \mathrm{a}^{*}}$, Dengjie $\mathrm{ZHU}^{1,2, \mathrm{~b}}$ and Zhaoyun $\mathrm{WU}^{3, \mathrm{c}}$ \\ ${ }^{1}$ School of Mechanical \& Electrical Engineering, Henan University of Technology, Zhengzhou, China \\ ${ }^{2}$ School of Mechanical \& Electrical Engineering, Wuhan University of Technology, Wuhan, China \\ wulihui@haut.edu.cn, bzdj1973@163.com, cwzhaoyun@163.com \\ *Corresponding author
}

Keywords: Semiconductor, Interbay, Object, Petri net, Model.

\begin{abstract}
To analyze the performance of automated Interbay material handling system in semiconductor wafer fabrication system, an Object-oriented and Colored Timed Petri Net (OCTPN) model was presented. The model could describe the practical hardware and operation considerations of Interbay system, such as transportation rail, shortcut, stocker and routing of overhead hoist transporters. To decrease complexity of modeling process, these practical hardware and operation considerations of Interbay system were encapsulated with object class. Therefore, the OCTPN model could be re-configurable and re-usable. With data from an Interbay material handling system of 200 $\mathrm{mm}$ semiconductor wafer fab, an illustrative example is built to evaluate the model. The result shows that the proposed OCTPN model is effective.
\end{abstract}

\section{Introduction}

The semiconductor wafer fabrication system (SWFS) distinguishes itself from traditional manufacturing systems by many characteristics, such as high reentrant flows, enormous process complexity, high level of work-in-process (WIP), shared production and testing tools, small batch production, long cycle times, and tight due dates [1]. These characteristics lead to high level of wafer lot (in wafer fabrication, a certain quantity of wafers is grouped in a standard container to transport, called a wafer lot) transportations within the semiconductor fabrication line [2]. Therefore, automated Interbay material handling system (often be called Interbay) becomes critically important to reduce manufacturing cost and maintain high tool utilization [3]. During the Interbay design process, to rapidly and effectively evaluate and analyze the performance of Interbay in SWFS, a practical and feasible Interbay model should be adopted.

Recently, there are many researches on performance analytical model for material handling system, such as queuing theory model [4], queuing network model [5], Markov chain model [6], and so on. However, these models are mainly suitable for simple closed-loop material handling system without shortcut rail. In addition, these models are difficult to concretely and accurately presented practical hardware and operations of Interbay system, which is critical important to evaluate the performance of Interbay system accurately and effectively. Petri net model has the capacity of modeling the MHS specifically and in detail [7]. However, for large-scale and complex MHS and SWFS, PN-based modeling approaches are easily to lead to model space explosion. In addition, the PN-based models are highly system dependent and lack re-configurability and re-usability. To make up these shortcomings, some object-oriented Petri nets model have been researched [8-9]. However, these object-oriented Petri nets models are difficult to effectively describe the overhead hoist transporter's routing operation in Interbay and to integrate with Interbay's dynamic scheduling approach, which has obviously influence on interbay's performance. To solve the problem, the paper proposes an objected-oriented and colored timed Petri net (OCTPN) model, in which Interbay's practical hardware and operation considerations, such as transportation rail, shortcut, stocker and routing of overhead hoist transporter, can be presented and encapsulated effectively. 


\section{Definitions of OCTPN Models}

Interbay is ofen consists of four kinds of hardware modules, including transportation rails, shortcuts, stockers and overhead hoist transporter (OHT). A detailed description of the Interbay operation can be found in reference [10].

To presents practical hardware and operation considerations of Interbay, an OCTPN modeling method is proposed. The OCTPN is defined as a 3-tuple structure, $K C T P N=\{O P S, T C, A\}$, where,

(1) $O P S=\left\{O P_{1}, \ldots, O P_{i}, \ldots O P_{n}\right\}$, which is the set of basic object places. $O P_{i}$ represent the hardware modules (such as transportation rails, shortcuts and stockers) place and logical module (such as routing) place in Interbay.

(2) $T C=\left\{T C_{1}, T C_{2}, \ldots T C_{j}, \ldots T C_{m}\right\}$, which corresponds to a limited set of communication transition, where $T C_{j}$ is the $j$ th communication transition that provides communication among object places. $O P_{i} \cup T C_{j} \neq \Phi, O P_{i} \cap T C_{j}=\Phi$.

(3) $A \subseteq I \cup O$, which is the sets of relationship function of object places OPS and communication transitions $T C$, where $I\left(O P_{i}, T C_{j}\right)$ is input function, and $O\left(A P_{i}, T C_{j}\right)$ is output function.

The interior behavior of the basic object place is encapsulated in a KCTPN model. An object place $O P_{j}$ is defined by a 5-tuple structure: $O P_{j}=\left\{P^{j}, T^{j}, C^{j}, A_{d}^{j}\right\}$. Where, (1) $P^{j}=\left\{L P^{j}\right\} U\left\{M P^{j}\right\} U\left\{S P^{j}\right\} . L P^{j}$ is a set of resource places to describe the interior operation states of hardware modules. $M P^{j}=\left\{I M^{j}, O M^{j}\right\}$ is a set of message places to communicate between objects place. (2) $T^{j}=\left\{T I^{j}, T S^{j}, T E^{j}\right\}$, which is a set of transitions, where $T I^{j}$ is the set of immediate transitions, $T S^{j}$ is a set of the stochastic transitions, and $T E^{j}$ is a set of the deterministically timed delay transitions. In addition, $P^{j} \cup T^{j} \neq \Phi, P^{j} \cap T^{j}=\Phi$. (3) $C^{j}$ corresponds to a color set of places and transitions in object place. $C^{j}\left(P^{j}\right)$ and $C^{j}\left(T^{j}\right)$ corresponds to respective colors sets of $P^{j}$ and $T^{j}$. (4) $A_{d}^{j}$ is a set of connecting arcs function between places and transitions, in which $\left\{L P^{j}\right.$ or $I M^{j}$ or $\left.S P^{j}\right\} \times\left\{T I^{j}\right.$ or $T S^{j}$ or $\left.T E^{j}\right\} \rightarrow\{0,1\}$ is input function and $\left\{T I^{j}\right.$ or $T S^{j}$ or $\left.T E^{j}\right\} \times\left\{L P^{j}\right.$ or $O M^{j}$ or $\left.S P^{j}\right\} \rightarrow\{0,1\}$ is output function.

\section{OCTPN Model for Interbay System}

In this paper, a simplified Interbay material handling system of 200mm SWFS (as shown in Fig. 1), which is an interbay with 22 stockers and 4 shortcuts, is used as the case to build the OCTPN model. Accord to the Interbay's hierarchical organization, OCTPN model of Interbay will be built in a hierarchical sequence. The modeling procedure includes following.

(1) Since transportation rails, shortcuts and stockers are independent modules on structure, OCTPN model of these modules is built and encapsulated respectively. The transportation rails, shortcuts and stockers model are built as shown in Fig. 2-Fig. 4., respectively.

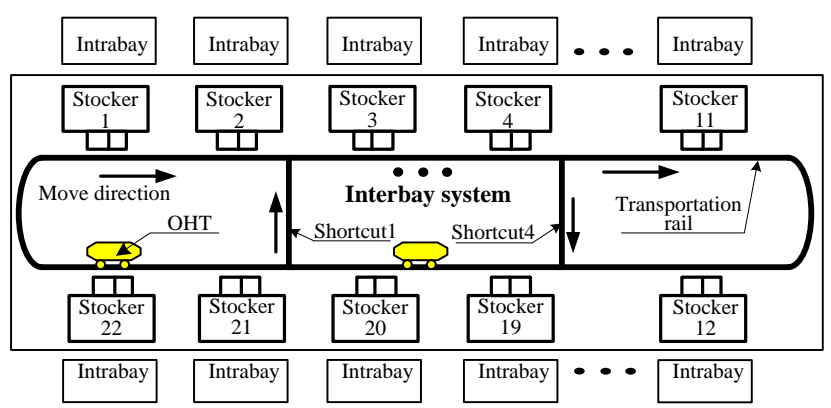

Figure 1. The layout of Interbay

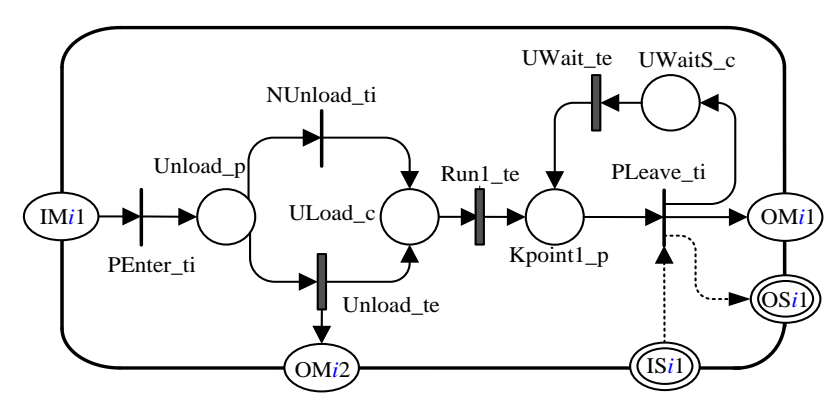

Figure 2. The OCTPN-based transportation rail model

(2) As OHTs run on transportation rails and shortcuts sequentially and interact with stockers to load and delivery wafer lots, OHTs and wafer lots can be modeled as different kinds of tokens respectively.

(3) Finally, integrated OCTPN model is constructed according to Interbay's infrastructure. The built OCTPN-based Interbay model is shown in Fig. 5. Detailed reasoning process of Interbay model 
is described as following. Firstly, vehicles run on transportation rails. When wafer lot token in stocker Stocker_ $i$ requests to move to destination stocker Stocker $j$, then the optimal empty vehicle is

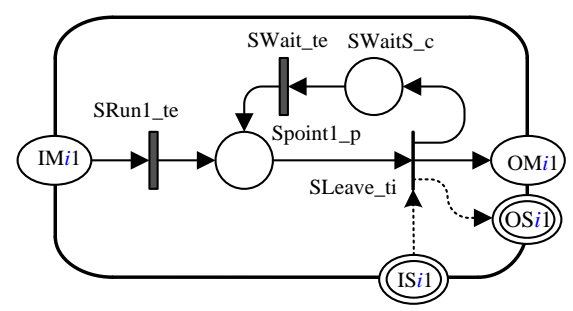

Figure 3. The OCTPN-based shortcut model

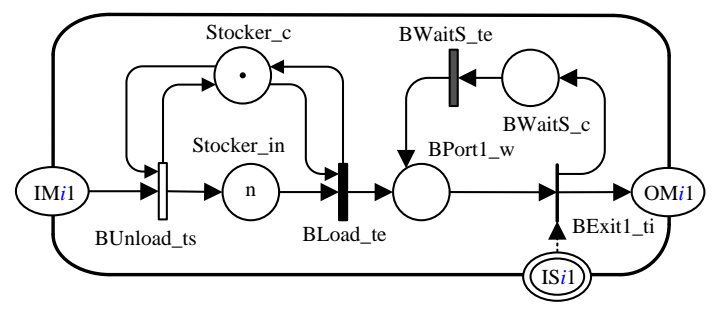

Figure 4. The OCTPN-based stocker model

designated to carry it based on the Interbay scheduling approach (such as first encounter first service (FEFS), highest priority first (HPF), and so on). After the designated vehicle token enters the transportation rail VPA_ap_i , then the communication transition $R K \_t i \_i$ and $S K \_t i \_i$ are fired. Next, the empty vehicle token in VPA_ap_i and wafer lot token in Stocker_i enter the transportation rail place $V P B \_a p \_i$, and the wafer in stocker is loaded by empty vehicle. After firing the communication transition $R R_{-} t i_{-} i$ and $C R \_t i$, the occupied vehicle token enters the route object place Route_ap . Guided by running path table of Interbay, the occupied vehicle token chooses to enter downstream transportation rail or shortcut until arriving at transportation rail VPA_ap_j. After that, the communication transition $S P_{-} t i_{-} j$ is fired and wafer lot token is unloaded to stocker object Stocker $\_j$. At the same time, the communication transition $R K_{-} t i_{-} j$ is fired and empty vehicle token enters downstream transportation rail object $V P B \_a p \_j$. The color sets of the Interbay object model are shown in Table 1.

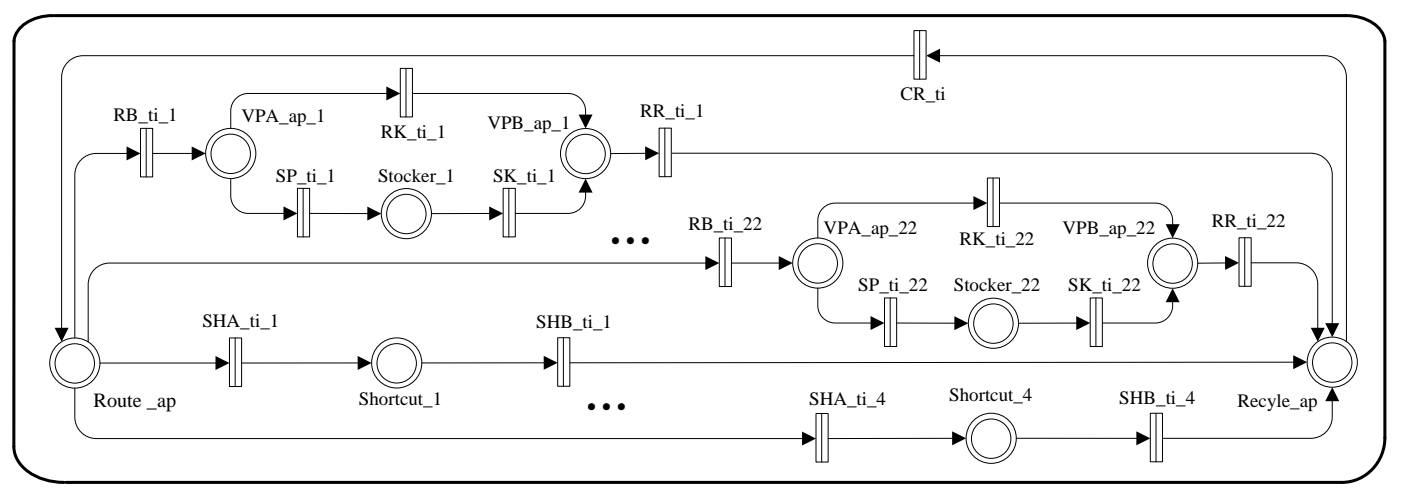

Figure 5. The OKCTPN-based Interbay model

Table 1. Color Set of The Interbay Model.

\begin{tabular}{|c|c|c|}
\hline Name & Function interpretation & Color set \\
\hline RB_ti_i & Vehicle enters the $i^{\text {th }}$ unloading transportation rail VPA_ap_i & $V T \times\left\{v_{e}\right\} \cup V T \times\left\{v_{w}\right\} \times L I \times\left\{S_{i}\right\}$ \\
\hline VPA_ap_i & Vehicle runs on the $i^{\text {th }}$ unloading transportation rail VPA_ap_i & $V T \times\left\{v_{e}\right\} \cup V T \times\left\{v_{w}\right\} \times L I \times\left\{S_{i}\right\}$ \\
\hline SP_ti_i & Wafer lot is unloaded by the vehicle located at VPA_ap_i & $L I \times\left\{S_{i}\right\}$ \\
\hline Stocker_i & Wafer lot be stored in/released from stocker & $L I \times\left\{S_{i}\right\}$ \\
\hline SK_ti_i & $\begin{array}{l}\text { Wafer lot is loaded by vehicle located at loading transportation rail } \\
V P B \_a p \_i\end{array}$ & $L I \times\left\{S_{i}\right\}$ \\
\hline VPB_ap_i & Vehicle runs on the loading transportation rail VPB_ap_i & $V T \times\left\{v_{e}\right\} \cup V T \times\left\{v_{w}\right\} \times L I \times\left\{S_{i}\right\}$ \\
\hline RK_ti_i & Vehicle enters the loading transportation rail VPB_ap_i & $V T \times\left\{v_{e}\right\} \cup V T \times\left\{v_{w}\right\} \times L I \times\left\{S_{i}\right\}$ \\
\hline RR_ti_i & Vehicle leaves current loading transportation rail VPB_ap_i & $V T \times\left\{v_{e}\right\} \cup V T \times\left\{v_{w}\right\} \times L I \times\left\{S_{i}\right\}$ \\
\hline SHA_ti_j & Vehicle enters the $j^{\text {th }}$ shortcut Shortcut $-j$ & $V T \times\left\{v_{w}\right\} \times L I \times\left\{S_{i}\right\}$ \\
\hline SHB_ti_j & Vehicle leaves the $j^{\text {th }}$ shortcut Shortcut $j_{-}$ & $V T \times\left\{v_{w}\right\} \times L I \times\left\{S_{i}\right\}$ \\
\hline Shortcut_ $j$ & Vehicle runs on the $j^{\text {th }}$ shortcut Shortcut $j_{-}$ & $V T \times\left\{v_{w}\right\} \times L I \times\left\{S_{i}\right\}$ \\
\hline
\end{tabular}




\begin{tabular}{|l|l|l|}
\hline CR_ti & Request of vehicle route & $V T \times\left\{v_{e}\right\} \cup V T \times\left\{v_{w}\right\} \times L I \times\left\{S_{i}\right\}$ \\
\hline Recyle_ap & Vehicle prepares to route & $V T \times\left\{v_{e}\right\} \cup V T \times\left\{v_{w}\right\} \times L I \times\left\{S_{i}\right\}$ \\
\hline Route_ap & Vehicle routes & $V T \times\left\{v_{e}\right\} \cup V T \times\left\{v_{w}\right\} \times L I \times\left\{S_{i}\right\}$ \\
\hline
\end{tabular}

\section{Conclusions}

The paper proposes a kind of OCTPN-based Interbay model, which describes the practical hardware and operation considerations of Interbay effectively, such as transportation rail, shortcut, stocker, routing of OHT, and so on. Since the OCTPN model is re-configurable and re-usable, the complexity of modeling can be simplified. With Interbay system data of a $200 \mathrm{~mm}$ semiconductor wafer fabrication line, the OCTPN-based Interbay model has been built. The performance indexes of different Interbay schedule approaches can be analyzed and compared based on the model.

\section{Acknowledgement}

This work was financially supported by the National Nature Science Foundation of China under Grant No. 71201048, Plan for Scientific Innovation Talent of Henan University of Technology No. 2014 CXRC02.

\section{References}

[1] R. Uzsoy, C.Y. Lee, and L.A. Martin-Vega. A review of production planning and scheduling models in the semiconductor industry, part I: System characteristics, performance evaluation and production planning. IIE Trans. Vol. 24 (1992), p. 47.

[2] T.C. Chiang. Enhancing rule-based scheduling in wafer fabrication facilities by evolutionary algorithms: Review and opportunity. Comp. \& Ind. Eng. Vol. 64 (2013), p. 524.

[3] J.T. Lin and C.J. Huang. A simulation-based optimization approach for a semiconductor photobay with automated material handling system. Simul. Model. Pract. Theory Vol. 46 (2014), p. 76.

[4] J.M. Smith. Robustness of state-dependent queues and material handling systems. Int. J. Prod. Res. Vol. 48 (2010), p. 4631.

[5] M. Jain, S. Maheshwarl, and K. Baghel. Queueing network modeling of flexible manufacturing system using mean value analysis. Appl. Math. Model. Vol. 32 (2008), p. 700.

[6] D. Nazzal and L.F. Mcginnis. Analytical approach to estimating AMHS performance in $300 \mathrm{~mm}$ Fabs. Int. J. Prod. Res. Vol. 45 (2007), p. 571.

[7] M.C. Zhou and M.D. Jeng. Modeling, analysis, simulation, scheduling, and control of semiconductor manufacturing systems: a Petri net approach. IEEE Trans. on Semicond. Manuf. Vol. 11 (1998), p. 333.

[8] H.R. Liu, Z.B. Jiang, and R. Fung. Modeling of large-scale complex re-entrant manufacturing systems by extended object-oriented Petri nets. Inter. J. Adv. Manuf. Tech. Vol. 27 (2005), p. 190.

[9] B.H. Zhou, Q.Z. Pan, S.J. Wang, et al.. Modeling of photolithography process in semiconductor wafer fabrication systems using extended hybrid Petri nets. J. Cent. South Univ. Tech. Vol. 14 (2007), p. 393.

[10] L.H. Wu, P.Y. Mok, and J. Zhang. An adaptive multi-parameter based dispatching strategy for single-loop interbay material handling systems. Comp. in Ind. Vol. 62 (2011), p. 175. 